\title{
Data-driven Prediction of Temperature Evolution in Metallic Additive Manufacturing Process
}

Thinh Quy Duc Pham, Truong Vinh Hoang, Quoc Tuan Pham, Than Phuc Huynh, Van Xuan Tran, Seifallah Fetni, Laurent Duchêne, Hoang Son Tran and Anne Marie Habraken

Thinh Quy Duc Pham. Thu Dau Mot University, Vietnam.

University of Liège, UEE Research Unit, MSM division, allée de la Découverte, 9 B52/3, B 4000 Liège, Belgium.

Truong Vinh Hoang. RWTH-Aachen University, Germany.

Quoc Tuan Pham. Division of Computational Mathematics and Engineering, Institute for Computational Science, Ton Duc Thang University, Vietnam.

Than Phuc Huynh. Thu Dau Mot University, Vietnam.

Van Xuan Tran. Thu Dau Mot University, Vietnam.

Corresponding author : xuantv@tdmu.edu.vn

Seifallah Fetni. University of Liège, UEE Research Unit, MSM division, allée de la Découverte, 9 B52/3, B 4000 Liège, Belgium.

Laurent Duchêne. University of Liège, UEE Research Unit, MSM division, allée de la Découverte, 9 B52/3, B 4000 Liège, Belgium.

Hoang Son Tran. University of Liège, UEE Research Unit, MSM division, allée de la Découverte, 9 B52/3, B 4000 Liège, Belgium.

Anne Marie Habraken. University of Liège, UEE Research Unit, MSM division, allée de la Découverte, 9 B52/3, B 4000 Liège, Belgium.

Fund for Scientific Research F.R.S-FNRS

\begin{abstract}
In this study, a data-driven deep learning model for fast and accurate prediction of temperature evolution and melting pool size of metallic additive manufacturing processes are developed. The study focuses on bulk experiments of the M4 high-speed steel material powder manufactured by Direct Energy Deposition. Under non-optimized process parameters, many deposited layers (above 30) generate large changes of microstructure through the sample depth caused by the high sensitivity of the cladding material on the thermal history. A 2D finite element analysis (FEA) of the bulk sample, validated in a previous study by experimental measurements, is able to achieve numerical data defining the temperature field evolution under different process settings. A Feed-forward neural networks (FFNN) approach is trained to reproduce the temperature fields generated from FEA. Hence, the trained FFNN is used to predict the history of the temperature fields for new process parameter sets not included in the initial dataset. Besides the input energy, nodal coordinates, and time, five additional features relating layer number, laser location, and distance from the laser to sampling point are considered to enhance prediction accuracy. The results indicate that the temperature evolution is predicted well by the FFNN with an accuracy of $99 \%$ within 12 seconds.
\end{abstract}

Keywords. Deep Learning, Temperature Evolution, Direct Energy Deposition

\section{Introduction}

Additive Manufacturing (AM) technology is a unique capability for building complex three-dimensional (3D) objects from computer-aided design models. Among many technologies used for metallic AM, Directed Energy Deposition (DED) is an interesting process that is flexible and adapted to repair operation. This method involves the deposition of metallic powder, which is melted via a focused heat source. DED is becoming widely used in industries such as aerospace [1], bio-design [2].

In order to identify optimal process parameters of AM, a design of experiments is often used [3]. However, performing the experiments of AM to find the optimal parameters is very expensive and time-consuming. The numerical approach, such as the Finite Element Method (FEM), is often employed to simulate the AM process [4]. However, the computing cost of these models remains excessively expensive when performing a large number of simulations. Therefore, it is 
Data-driven Prediction of Temperature Evolution in Metallic Additive Manufacturing Proc...

not suitable to directly conduct uncertainty quantification and optimization of process parameters using these models to achieve a robust solution. To overcome this challenge, Machine Learning (ML) techniques are employed to construct surrogate models representing the complex relations between the process parameter and the temperature history defining the part quality [5]. Thanks to the predictive ML-based surrogate models, the simulations can be performed with a negligible computational cost. Recently, the application of ML to the AM field received significant attention from both the industrial and academic sectors $[6,7,8]$. A comprehensive review of this application can be found in [5].

In AM process, many physical phenomena occur at a short period of time and at a temperature above the melting point of materials. These temperature profiles strongly affect material properties related to the generated microstructures. Some previous studies were performed to develop the ML-based surrogate model to predict the temperature evolution of the AM process. For instance, the Recurrent Neural Network (RNN) was developed to compute the temperature field for an arbitrary geometry with different scanning strategies 6]. Similarly, the temperature field is also predicted by the surrogate model with Bayesian loss function [7]. In addition, in [8], the temperature field was predicted directly by the Physics-Informed Neural Networks (PINNs) without numerical data.

The above ML-based models [7-9] are somewhat complicated (RNN, Bayesian) and only applicable for a few layers. Thus, it is essential to develop a simple ML-based model to directly predict the temperature field of the AM processes with a large number of layers. Based on this review, this study aims to develop a simple ML-based surrogate model to predict the temperature evolution as well as the melting pool size of a DED process of a cubic part with 36 layers. In this article, the data used to train the ML-based model are first generated using the Finite Element (FE) model, which has been validated with experimental data (see Section 2). In Section 3, the ML-based surrogate model is described with its results to predict the temperature evolution and melting pool size during the AM process.

\section{ML-based surrogate model for the DED process}

In this section, we describe the predictive ML-based model called also the surrogate model to predict the temperature evolution of the DED process. It is built using the following two-step process:

(i) Data collection and data pre-processing,

(ii) Evaluation of the surrogate model parameters.

For step (i), it is very important that the training data is physically representative. Note that this study focuses on bulk experiments of the M4 high-speed steel material powder. The material properties can be found in detail in [9]. Hereafter, the training data is generated by thermal simulations performed with the updated Lagrangian FE code "Lagamine" developed by ArGEnCo Department of the University of Liège [4], Belgium. The convection and radiation boundary conditions are applied as well as the birth element technique to model the process. The classical conduction non-linear equation is reminded as

$$
\frac{\partial}{\partial x}\left(k \frac{\partial T}{\partial x}\right)+\frac{\partial}{\partial y}\left(k \frac{\partial T}{\partial y}\right)+\frac{\partial}{\partial z}\left(k \frac{\partial T}{\partial z}\right)+Q_{i n t}=\rho c_{p} \frac{\partial T}{\partial t}
$$

where $T, k, Q_{i n t}, c p, \rho$ and $t$ are the transient temperature, thermal conductivity, the power generated per volume in the workpiece, apparent heat capacity, density and time, respectively. Fig. 1 plots and compares the temperature evolutions at one thermocouple located in the substrate obtained within the experiment and by the 2D FE simulation. The detailed description as well as the schematic of the DED experiment can be find in [4]. As shown in Fig. 1, the result of the 2D FE simulation (representative of the middle track of each layer) is in good agreement with the experimental bulk result. 
Consequently, the FE model is able to provide high-quality structure data to the ML-based surrogate models described hereafter.

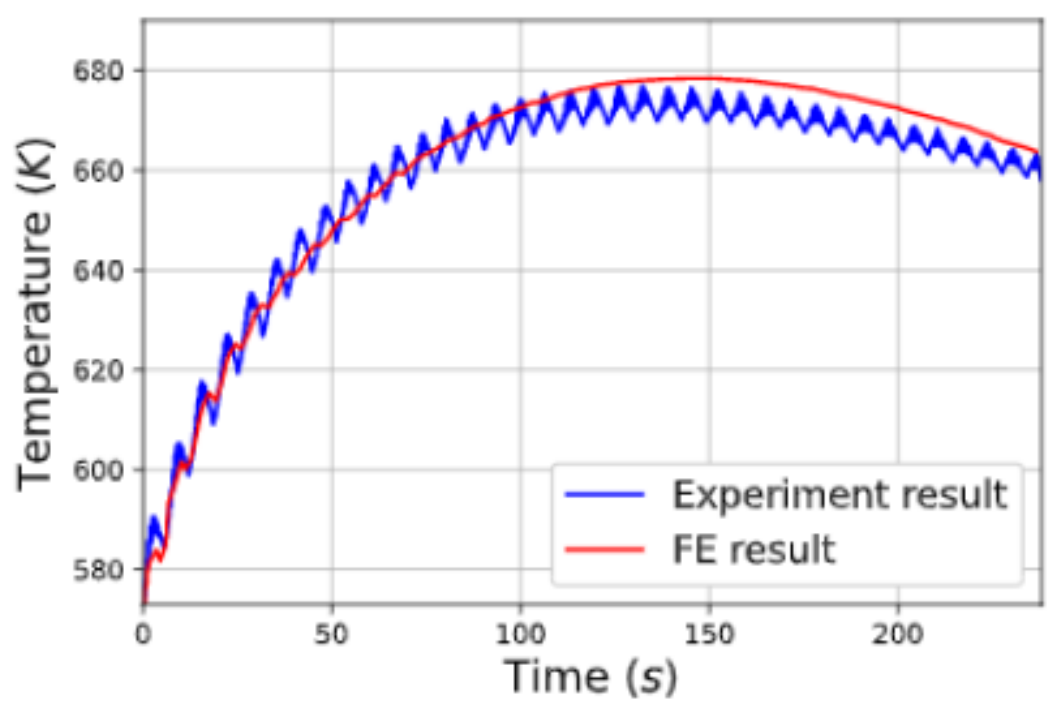

Fig. 1: The temperature evolution at one thermocouple of the experimental and FE model.

The dataset used in this study consists in five groups of data. Each group is the data obtained from one FE simulation with a value of input energy $Q_{i n t}$ (see Eqn. 1). The five values of $Q_{i n t}$ are chosen as $0.8 Q_{0}, 0.9 Q_{0}, 1.0 Q_{0}, 1.1 Q_{0}, 1.2 Q_{0}$, in which $Q_{0}=Q_{\text {reference }}=1 \mathrm{~W} / \mathrm{m}^{3}$. The data group obtained from $Q_{i n t}=1.0 Q_{0}$ is used for further validation and the remaining four data groups are used for training.

Each data group contains 4.8 million data points (see details below). Consequently, a total of 19.9 million data points is used for the training of the FFNN-based model. For step (ii), a FFNN is chosen as it has advantages in approximating highly non-linear and high-dimensional functions. However, training the FFNN with such a small dataset might lead to the over-fitting problem. As a consequence, the training dataset is partitioned into a training dataset and a validation dataset. Beside the input energy, nodal coordinates and time, five additional features are considered as input features to boost the performance of the FFNN-based model including the laser head location in $\mathrm{x}$ - and $\mathrm{y}$-direction, the distance from each sampling point to the laser head in $\mathrm{x}$ - and $\mathrm{y}$-direction and the current printing layer at each time-step. Note that these additional features are also used in the work of Fetni et. al [11]. For any point of interest, the following 9 features are defined (see Fig. 2).

(i) The input energy (Qint)

(ii) x-nodal coordinate (xp)

(iii) y-nodal coordinate (yp)

(iv) Time

(v) The laser head position at $\mathrm{x}$-coordinate $(\mathrm{xl})$

(vi) The laser head position at y-coordinate (yl) 
Data-driven Prediction of Temperature Evolution in Metallic Additive Manufacturing Proc...

(vii) The distance from laser head to each sampling point in $\mathrm{x}$-direction $(\mathrm{dx})$

(viii) The distance from laser head to each sampling point in y-direction (dy)

(ix) The number of the current printing layer

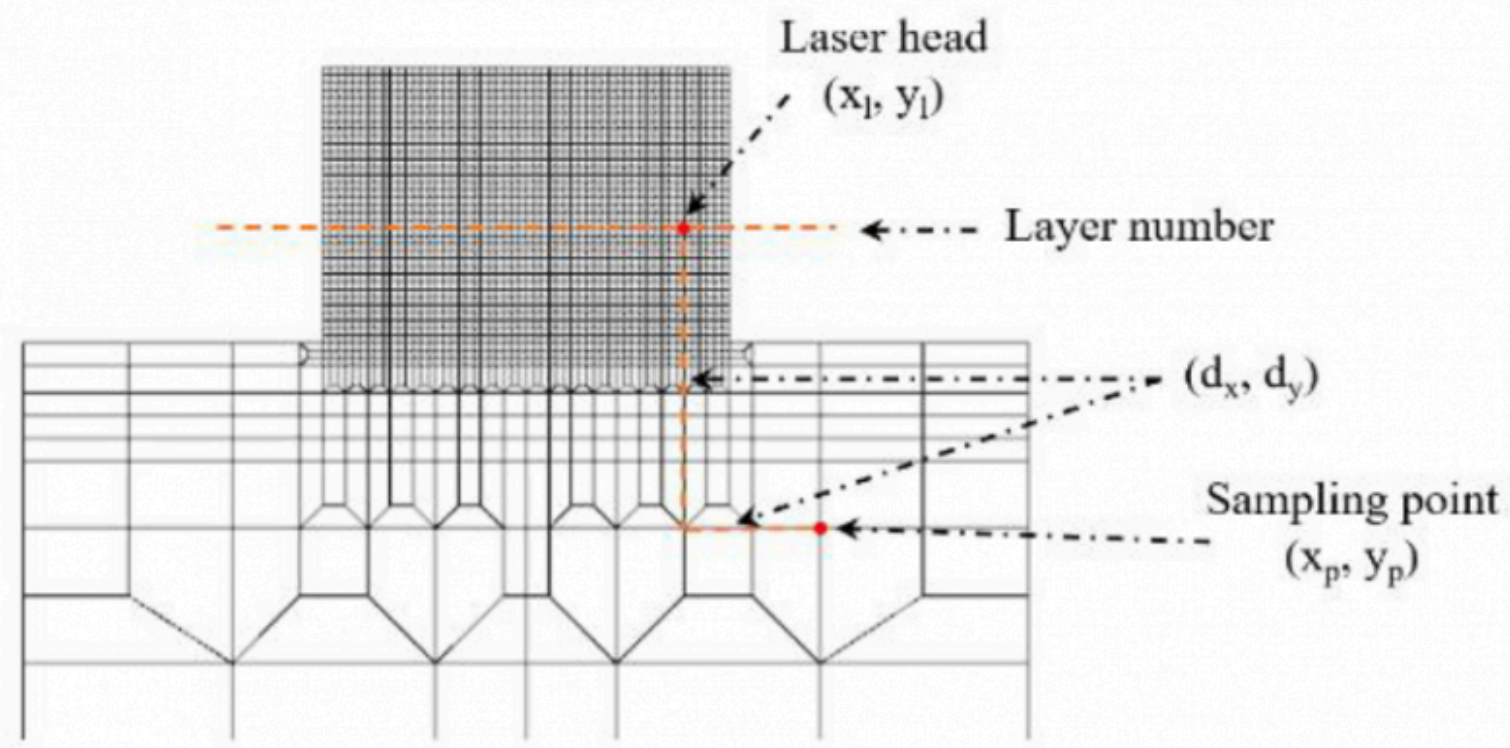

Fig. 2: The input features of the surrogate model

Overall, the input data consists in $\left\{\mathbf{x}_{j}^{(i)} ; i=1, \ldots, N ; j=1, \ldots, 9\right\}$, where $N$ is the number of configurations. Note that $N=19.9$ million (2519 nodes $\times 1978$ time-step $\times 4$ simulation data) configurations as described above. The FFNN-based model is trained by optimizing the weights and biases $W$ that exist inside the model. It is done by solving the mean squared error (MSE) problem for each iteration:

$$
\begin{gathered}
\boldsymbol{W}=\underset{\boldsymbol{V}}{\operatorname{argmin}} \mathcal{L}(\boldsymbol{V}), \\
\mathcal{L}(\boldsymbol{V})=\frac{1}{N_{T}} \sum_{i=1}^{N_{T}}\left(f\left(x^{(i)} \mid \boldsymbol{V}\right)-T^{(i)}\right)^{2},
\end{gathered}
$$

where, $\mathcal{L}(\boldsymbol{V}), N_{T}$ and $T^{(i)}$ are the MSE loss function, training data and the temperature value corresponding to each configuration, respectively. Note that $\boldsymbol{V}$ is the matrix of weights to be optimized, argmin is the argument of minimum and $N_{T}=30 \% N$ is chosen for this task. The Adaptive Moment Estimation [10] algorithm is used in the stochastic gradient descent procedure to update network weights after each iteration based on training data with the learning rate of 0.001. In addition, to assess the performance of FFNN-based model, the metric of the coefficient of determination $R^{2}$ is used. It is defined as 


$$
R^{2}=1-\frac{\sum_{i=1}^{M}\left(\hat{T}^{(i)}-T^{(i)}\right)^{2}}{\sum_{i=1}^{M}\left(\bar{T}-T^{(i)}\right)^{2}},
$$

where, $M, \hat{\mathrm{T}}^{(\mathrm{i})}, T$ and $\mathrm{T}^{(\mathrm{i})}$ are the number of samples, the predicted temperature from the FFNN-based model, mean temperature and actual temperature obtained by 2D FE model, respectively. Hence, the closer to 1 the value of $R^{2}$ is, the better the model predicts.

\section{Results obtained from FFNN-based model}

This section presents the prediction results obtained by the FFNN-based model. The analysis of the temperature results is based on three important points including the substrate $(S)$ and the cladding $(P)$ as shown in Fig. 3. Note that the cladding points $\mathrm{P}_{1}$ and $\mathrm{P}_{2}$ are located on the symmetric line of the component.

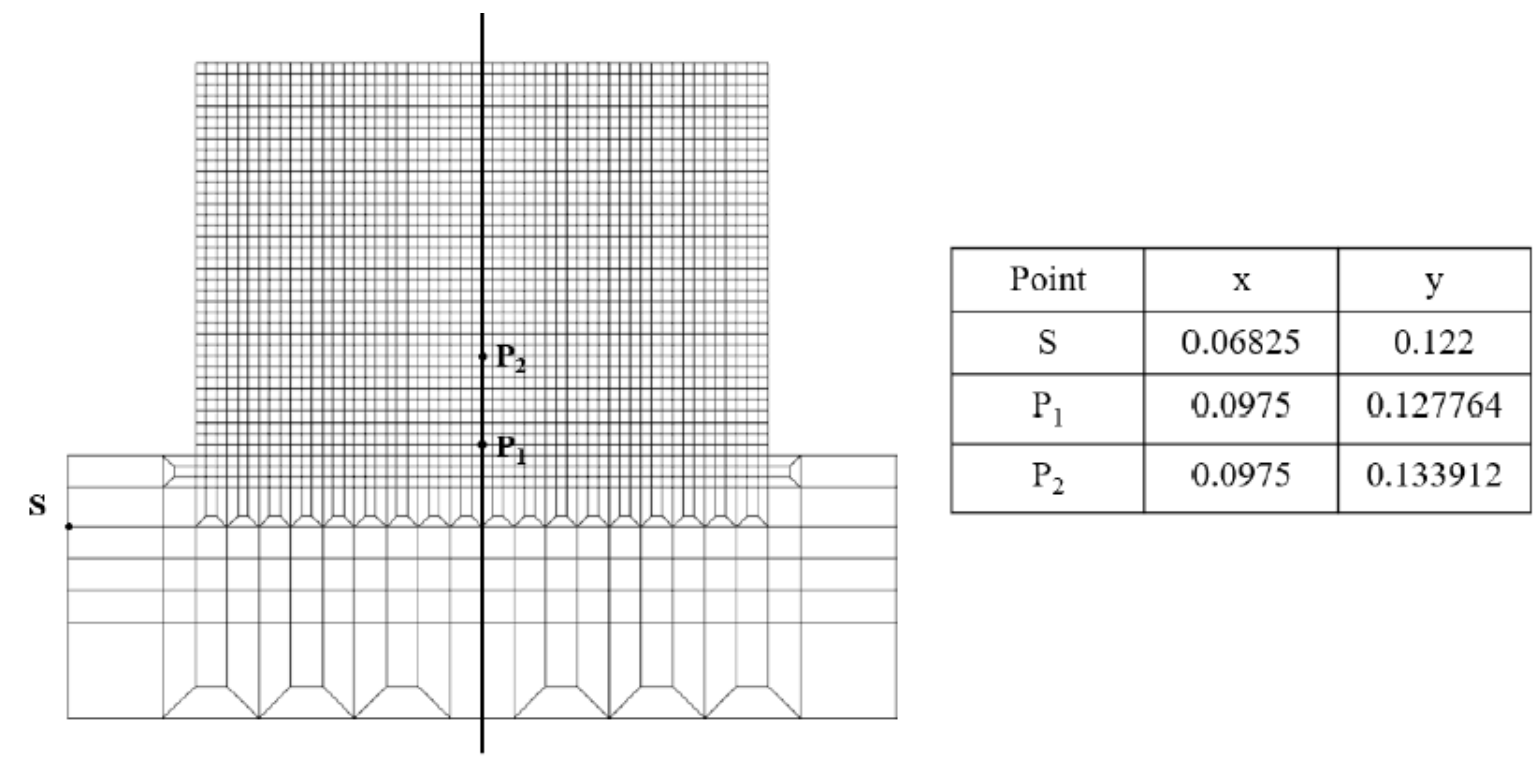

Fig. 3: Position of three interest points at which temperature evolution is examined

Fig. 4 shows the train and validation losses of the model. It is observed that the training process ends at 300 epochs as the validation loss does not further decrease. Note that the epoch is the optimization step, Loss is the training loss per each epoch (see Fig. 4), Val_Loss is the validation loss per each epoch (see Fig. 4). The converged value of the validate set is chosen to correspond to a MSE lower than $5 \times 10^{-5}$ as it is acceptable for the problem (see Fig. 4). 


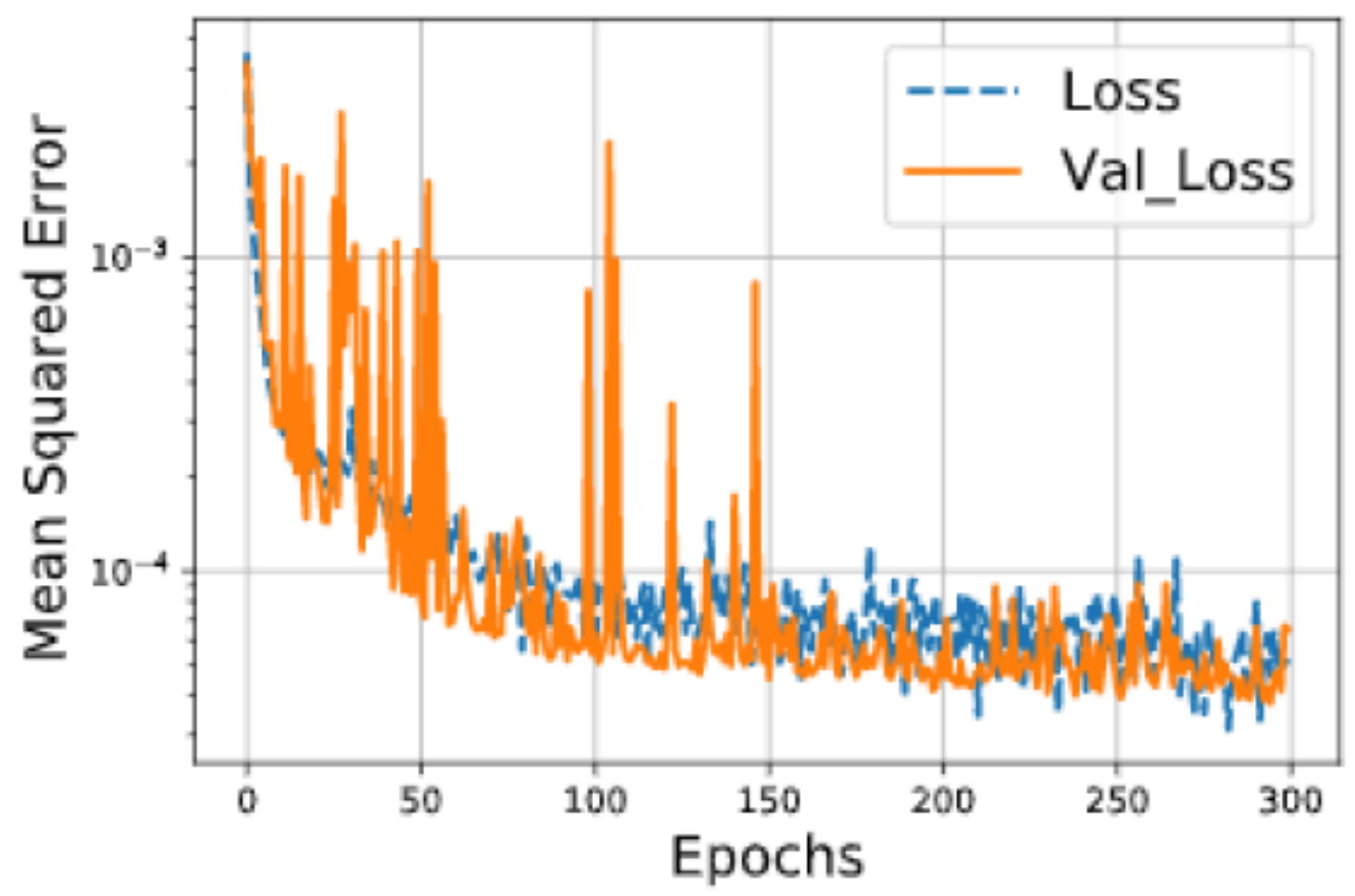

Fig. 4: Training and validation loss of the FFNN-based model at each optimization step

\subsection{Prediction of the temperature evolution of the DED process}

Fig. 5 shows the temperature evolution for three interest points, namely $\mathrm{S}, \mathrm{P}_{1}$, and $\mathrm{P}_{2}$ representative of the substrate and two different layers in the printed part. At each point, the temperature profile shows the characteristic cyclic thermal history related to the position of the laser head.

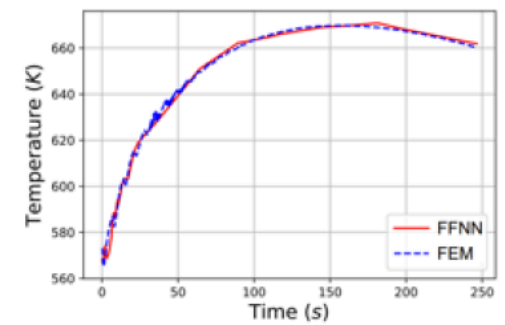

(a)

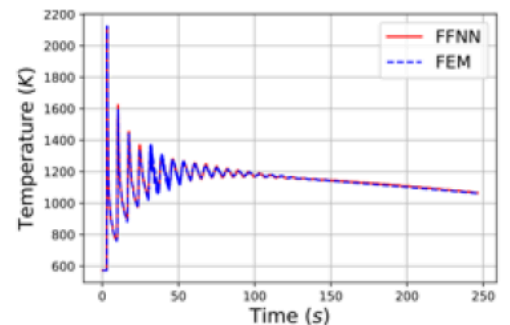

(b)

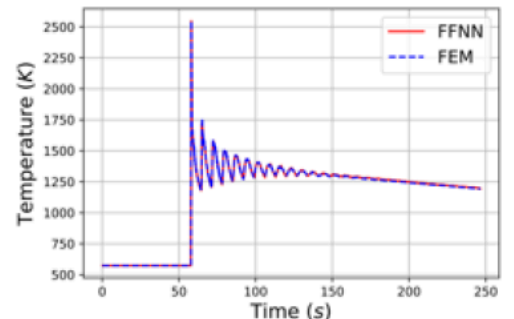

(c)

Fig. 5: Temperature evolution predicted at 3 locations (a) substrate S, (b) Cladding P1 and (c) Cladding P2 by FE and FFNN-based models

For the temperature evolution of the substrate S (see Fig. 5(a)), the result shows a good agreement between the temperature profile computed by the FFNN-based model and the FE model. It is noted that the 5 additional features named (v) to (ix) in Fig. 2 are set to zero for all the substrate points. This choice is explained by the observation that the substrate point $\mathrm{S}$ stays far from the laser head and its temperature value is not affected much by these additional features. Consequently, the prediction of the substrate point is just a function of the nodal coordinates and time. In 
detail, the substrate point has a R2 value of 0.995. Fig. 5(b) and Fig. 5(c) show the comparison of the temperature evolution of the cladding $\mathrm{P}_{1}$ and $\mathrm{P}_{2}$ obtained from FE and FFNN-based models. Similar to substrate $\mathrm{S}$ point, the temperature evolution of the two cladding points is predicted well by the FFNN-based model with a high $\mathrm{R}^{2}$ value of 0.991 and 0.999, respectively. As shown in Fig. 5, the oscillations of the temperature profiles as well as the temperature peaks are well captured by the FFNN-based model. Table 1 shows the computational cost and output data size of the FE and FFNN-based models. As observed in Table 1, the time required to obtain the temperature history of the FEM simulation for 4540 finite elements is reported as 1800 seconds. On the other hand, the FFNN-based model only takes 12 seconds to get the results. In summary, the FFNN-based model outperformed the FE model in computing time once datasets and FFNN-based model are developed.

Table 1. Computational cost of FE and FFNN-based models

\begin{tabular}{ccc}
\hline \multirow{2}{*}{$\begin{array}{c}\text { Number of } \\
\text { elements }\end{array}$} & \multicolumn{2}{c}{ Execution time } \\
\cline { 2 - 3 } & FEM (s) & FFNN (s) \\
\hline 4540 & 1800 & 12 \\
\hline
\end{tabular}

\subsection{Prediction of the melting pool size}

Fig. 6 shows the comparison of the melting pool size obtained from the FE and FFNN-based models. The melting pool size is the liquid zone generated by the laser. It is built by the powder flow melted by the laser energy as well as by the fusion of the previous build layers. The size of the melting pool plays an important role in determining the microstructure and mechanical properties of the printed sample. It is directly extracted from the temperature field data as the material points having a temperature higher than the melting temperature. As shown in Fig. 6, the predicted melting pool size is in good agreement with FE prediction with a $\mathrm{R}^{2}$ value of 0.971 . It is noted that the value of melt pool equal to zero means the laser is switched off. Similar to the temperature field prediction, the peak variations of the melting pool area with the height of the cladding are well captured by the FFNN-based model. 


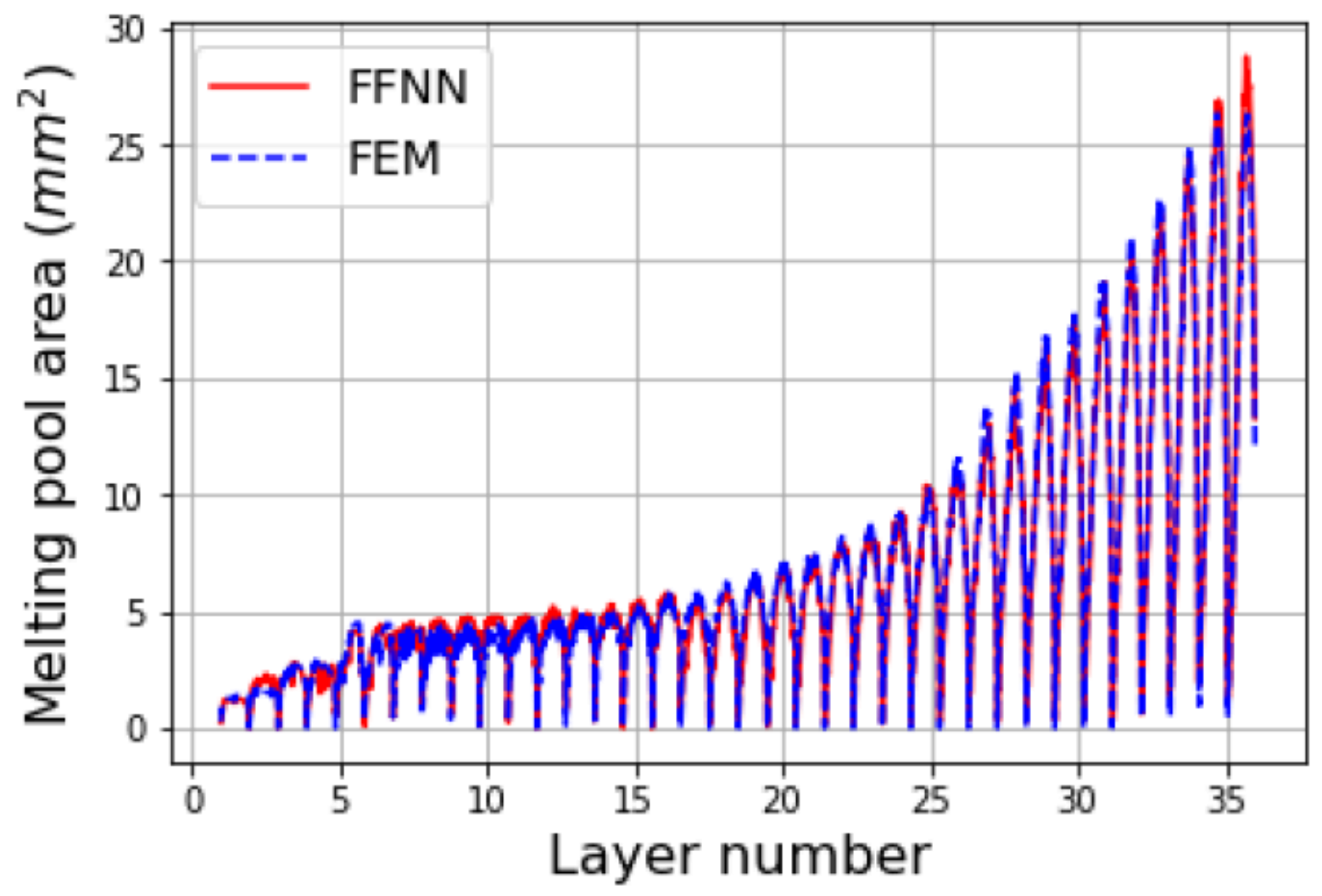

Fig. 6: Melting pool area predicted from FE and FFNN-based models

\subsection{Assessment of the FFNN-based model prediction}

In this section, the assessment of the FFNN-based model prediction is performed to give an insight into the model performance. Given a very small amount of FEM simulation data, one needs to assess the predictive ability of the model compared with the case of a larger amount of FEM simulation data. Each FEM simulation data is created by changing the value of input energy $Q_{\text {int }} \in[0.8,1.2]\left[Q_{0}\right]$. At this stage, a total of 18 FEM simulation data is created instead of the 5 groups of Section 2, and then they will become the validation data of the FFNN-based model. The value of Qint used to create FEM simulation data for training and validation of the FFNN-based model are described in Table 2. As shown in Fig. 7, the model predicts the other 18 FEM simulation data with a value of $\mathrm{R}^{2}$ greater than 0.99 while the FFNN-based model is trained by only 5 FEM simulation data (see Table 2). Note that the datasets used in training are also used for validation. Accordingly, the FFNN-based model is able to predict the FEM simulation data created by the input energy $Q_{\text {int }} \in[0.8,1.2]\left[Q_{0}\right]$ with excellent accuracy.

Table 2. The value of $Q_{\text {int }}$ used to create FEM simulation data for training and validation of the FFNN-based model

\begin{tabular}{|c|c|c|c|c|c|c|c|c|c|c|c|c|c|c|c|c|c|c|}
\hline $\mathrm{Q}_{\text {int }}\left(\mathrm{Q}_{0}\right)$ & 0.8 & 0.82 & 0.86 & 0.9 & 0.93 & 0.95 & 0.96 & 0.97 & 0.99 & 1 & 1.07 & 1.08 & 1.09 & 1.1 & 1.12 & 1.17 & 1.19 & 1.2 \\
\hline Training & $x$ & & & $x$ & & & & & & $x$ & & & & $x$ & & & & $x$ \\
\hline Validation & $x$ & $x$ & $x$ & $x$ & $x$ & $x$ & $x$ & $x$ & $x$ & $x$ & $x$ & $x$ & $x$ & $x$ & $x$ & $x$ & $x$ & $x$ \\
\hline
\end{tabular}




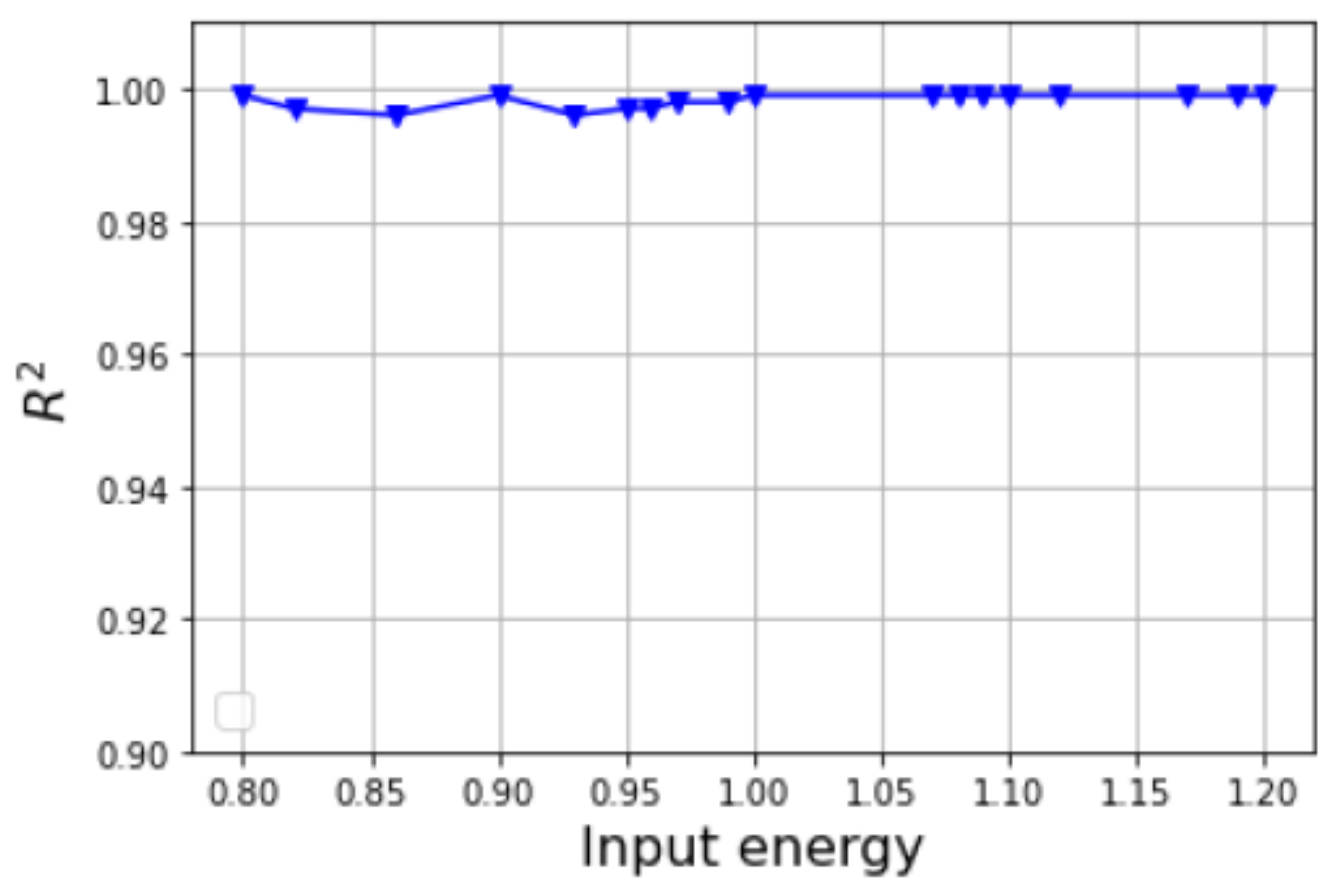

Fig. 7: Assessment of the FFNN-based model prediction using $\mathrm{R}^{2}$ metric

\section{Conclusion}

In this study, a simple FFNN-based surrogate model for the prediction of the temperature evolution and melting pool size in the DED process is developed. The numerical data of the evolution of the temperature fields under different process settings are obtained using a high-fidelity finite element model, which has been validated by experimental measurements. Beside the input energy, nodal coordinates and time, five additional features are considered as the input features of the FFNN-based model. Consequently, the surrogate model predicts the temperature evolution as well as the melting pool size of the DED process with excellent accuracies of $99 \%$ and $97 \%$, respectively. In the future study, an optimization framework for the process parameters will be developed using Bayesian optimization.

\section{Acknowledgements}

This work was funded by Vingroup and supported by Vingroup Innovation Foundation (VINIF) under project code VINIF.2020.DA15..

\section{Bibliography}

[1] Uriondo, A., Esperon-Miguez, M., \& Perinpanayagam, S. (2015). The present and future of additive manufacturing in the aerospace sector: A review of important aspects. Proceedings of the Institution of Mechanical Engineers, Part G: Journal of Aerospace Engineering, 229(11), 2132-2147.

[2] Culmone, C., Smit, G., \& Breedveld, P. (2019). Additive manufacturing of medical instruments: A state-of-the-art review. Additive Manufacturing, 27, 461-473.

[3] Mohamed, O. A., Masood, S. H., \& Bhowmik, J. L. (2015). Optimization of fused deposition modeling process parameters: a review of current research and future prospects. Advances in Manufacturing, 3(1), 42-53. 
Data-driven Prediction of Temperature Evolution in Metallic Additive Manufacturing Proc...

[4] Jardin, R. T., Tchuindjang, J. T., Duchêne, L., Tran, H. S., Hashemi, N., Carrus, R., ... \& Habraken, A. M. (2019). Thermal histories and microstructures in Direct Energy Deposition of a High Speed Steel thick deposit. Materials Letters, 236, 42-45. [5] Meng, L., McWilliams, B., Jarosinski, W., Park, H. Y., Jung, Y. G., Lee, J., \& Zhang, J. (2020). Machine Learning in Additive Manufacturing: A Review. JOM, 1-15.

[6] Mozaffar, M., Paul, A., Al-Bahrani, R., Wolff, S., Choudhary, A., Agrawal, A., ... \& Cao, J. (2018). Data-driven prediction of the high-dimensional thermal history in directed energy deposition processes via recurrent neural networks. Manufacturing letters, 18, 35-39.

[7] Ren, K., Chew, Y., Zhang, Y. F., Fuh, J. Y. H., \& Bi, G. J. (2020). Thermal field prediction for laser scanning paths in laser aided additive manufacturing by physics-based machine learning. Computer Methods in Applied Mechanics and Engineering, 362, 112734

[8] Roy, M., \& Wodo, O. (2020). Data-driven modeling of thermal history in additive manufacturing. Additive Manufacturing, 32, 101017.

[9] Hashemi, S. N. (2017). Study of High Speed Steel deposits produced by Laser cladding, Microstructure-WearThermal model (Doctoral dissertation, University of Liège, Liège, Belgium).

[10] Kingma, D. P., \& Ba, J. (2014). Adam: A method for stochastic optimization. ar Xiv preprint arXiv:1412.6980.

[11] Fetni, S., Pham, Q., Tran, V., Duchêne, L., Tran, H. \& Habraken, A. (2021) Thermal field prediction in DED manufacturing process using Artificial Neural Network. Paper presented at ESAFORM 2021. 24th International Conference on Material Forming, Liège.

PDF automatically generated on 2021-05-20 05:39:41

Article url: https://popups.uliege.be/esaform21/index.php?id=2599

published by ULiège Library in Open Access under the terms and conditions of the CC-BY License (https://creativecommons.org/licenses/by/4.0) 